\title{
Breast Rhabdomyosarcoma
}

National Cancer Institute

\section{Source}

National Cancer Institute. Breast Rhabdomyosarcoma. NCI Thesaurus. Code C5190.

A malignant mesenchymal tumor with skeletal muscle differentiation affecting the breast. 